\title{
The OpenMI 2.0 Standard for Integrating Numerical Models
}

\author{
Harpham, Q.K (HR Wallingford); Hughes, A. (BGS) and Moore R.V.
}

\begin{abstract}
The purpose of this paper is to introduce, explain and promote the Open Modelling Interface (OpenMI) version 2.0 standard for coupling environmental numerical models (simulations of environmental processes). It is intended to be accessible to readers of all levels of experience. During recent decades it has been recognised that the environment is made up of a complex set of interconnected processes. Therefore, understanding it requires not only understanding of the processes in isolation, but also the interactions between these processes. Traditional methods of simulating such environmental interactions have included passing the outputs of one numerical model into another or creating a single 'super-model' covering a variety of processes. OpenMI provides a standard method which could be applied to independent numerical model components and allow them to exchange data so that they could interact and influence one another. This is achieved without fundamental changes to the core of the components themselves.
\end{abstract}

\section{Keywords}

Open Modelling Interface

OpenMI

Integrated modelling

Model linking

Model coupling

Decision support systems

Open standards

Open source

Open Geospatial Consortium

\section{Software Availability}

The paper definition of OpenMI can be obtained from its associated OGC (Open Geospatial Consortium) standard definition at https://www.opengeospatial.org/standards/openmi.

Object interface libraries for OpenMI 2.0 standard can be obtained from its associated Source Forge project, https://sourceforge.net/projects/openmi/. They have been prepared in Java and C\#, together with other supporting material.

The OpenMI Association website is available at http://openmi.org. 
HR Wallingford's FluidEarth implementation of OpenMI can be obtained from its associated Source Forge project, https://sourceforge.net/projects/fluidearth/. A comprehensive training website with example numerical model compositions is available at http://eLearning.fluidearth.net.

\section{Introduction}

\section{Purpose}

The purpose of this paper is to introduce, explain and promote the appropriate use of the Open Modelling Interface (OpenMI) version 2.0 standard for coupling numerical models. It is intended to be accessible to readers of all levels of experience, from those writing and implementing numerical models through to those exploring the new discipline of integrated modelling for the first time. The paper supplements the full specification of the standard which was developed and is maintained by the OpenMI Association (OA) and is published by the Open Geospatial Consortium (OGC) (OGC OpenMI, 2013) as an OGC standard. It is an open standard and is available through the OGC website.

The OpenMI standard was developed in order to facilitate the understanding of interacting environmental processes. While it was conceived within the domains of hydraulics and hydrology, it was appreciated from the outset that the need was for a mechanism that could link across domain or disciplinary boundaries. Indeed, the OpenMI user community already extends well beyond the original domains and, indeed, beyond those concerned with simulating the natural environment. However, it was the growing need to understand the interconnectedness of environmental events and processes which prompted its inception.

\section{OpenMI 2.0 in Context}

Traditionally, environmental simulation models have been created by scientists working in independent specialist groups. Today, they nearly all take the form of computer programs and are referred to collectively as numerical models. These models usually consist of an algorithm that simulates one or a few specific processes articulated in computer code. The program receives the description of the situation it is to simulate from input data files and/or a user interface. Results from the simulation are usually returned via one or more output data files.

During recent decades it has been recognised that the environment is made up of a complex set of interconnected processes (ref Moore, Harpham etc.). Changes to one environmental phenomenon at one location can have a fundamental effect on other phenomena at another location (Meiburg 2007). As a result, environmental science is being challenged to address increasingly complex questions, such as "will the predicted rise in temperature due to climate change lead to Malaria being a health problem in more countries?" (Moore and Hughes, 2017). The traditional method of simulating such environmental interactions has been to run the process models separately, one after the other, the results of the first becoming the input of the second. (see Anastas, 2010 and also Voinov, 2010). For example, in order to simulate a flooding scenario, one numerical model simulating rainfall passes its output into another numerical model which understands river catchment drainage using rainfall as its input. A chain of models is constructed with the output of the 'upstream' model simply requiring interpolation or re-formatting in order for it to be made compatible with its 'downstream' counterpart.

By combining different models together the end result can answer complex questions more effectively than by using a single existing model. Further, the solution of complex questions require holistic solutions drawn from different disciplines (Laniak et al., 2013). Therefore, it may be 
preferred to combine numerical models together into one, larger, more comprehensive implementation. So instead of passing the rainfall between the model producing rainfall and the model receiving the rainfall data, the computer codes are combined into a single numerical model which performs both modelling functions. Internally, the same function will be being performed, but the passing of the rainfall will occur 'in memory' as the combined models execute. This second option opens up the possibility of allowing the processes to influence one another as they proceed through time. It may be that the phenomena being described are closely interlinked and having a two-way impact on each other. For example, if a torrent of water rushes against the side of the building then the presence of the building will have an effect on the passage of the water. The water may cause a wall of the building to collapse and so the presence of the water will affect the building. To correctly simulate this, the numerical model describing the state of the building structure will need to be combined with that describing the flow of the water. However, it is likely that the numerical model describing the flow of the water will come from one community of experts and the model describing the building structure will come from another. There may be no appetite to combine these into a single 'super-model'. Further, such a combined model will be exponentially more difficult to maintain and the freedom of the modelers to innovate will be severely inhibited. Thus, if these two model codes could be kept separate yet enabled to exchange data as they ran in parallel, behaving as a single composition, then each community could develop and innovate their own model without reference to the other.

It was recognised that considerable investment had been made into developing many numerical models by the organisations responsible for them. This investment couldn't simply be discarded in favour of developing a new generation of environmental numerical models from scratch. Therefore a solution which enabled model integration was sought which could be applied to any numerical model, irrespective of technology, history or ownership - a solution which respected intellectual property and diversity.

\section{The Case for Standardisation}

Users have required something more capable than manual data transfer between models and something more manageable and sustainable than 'super-models'. To this end, some numerical model developers have joined two or more established models together, keeping the core of each broadly separate but operating them together. For example, Sophocleous et al. (1999) demonstrate a combined MODFLOW-SWAT model combination where MODFLOW simulates groundwater and SWAT surface water. Other practitioners use web processing services to pass data between linked model components via the internet as illustrated by Nativi et al. (2013). The original intention behind OpenMI was to provide a standard method which could be applied to independent numerical model components and allow them to exchange data whilst they are running. This was to be achieved without fundamental changes to the core of the components themselves thus still allowing them to be run independently, when required.

This situation allows experts to remain within their domains of expertise, each continually innovating to produce successive generations of numerical model programs which increasingly accurately describe the phenomena they represent. Very often, such changes do not alter the data types taken in or output by the model. The changes therefore make no demands on the authors of other linkable models. Achieving this successfully depends on the interface between the models being tightly defined and that definition being readily accessible by all. Such common understanding drives the need for standardisation, thereby allowing such compositions to be built and executed correctly. Thus, each set of experts is required to engineer their software according to a specified standard. This standard would have to contain all the elements necessary for the inter-model communication, 
yet allow each set of domain experts the freedom to apply their own numerical implementation of the associated mathematical theories, as well as the necessary functional elements to achieve an accurate simulation.

It could be suggested that asking different domain experts to adopt a common standard for software engineering would hamper creativity. If they are restricted in how they can implement their numerical models then surely that restriction would hamper further experimentation and discovery? The authors submit that the opposite would be the case. Consider the example of music and musical notation. The decision to limit musicians from a continuous distribution of an infinite number of tones to just twelve notes - the seven natural notes plus five sharps and flats, notwithstanding the implementation of this across multiple octaves - serves to enable creativity. Having described the twelve notes, musicians then discovered that they could be combined to articulate chords as well as describing patterns and scales. They then created the common language of musical notation to communicate this. These processes enabled creativity rather than hampering it. It would seem absurd now to abandon this and revert entirely to music with no fixed notes or notation. Such is also the case for numerical modelling.

\section{The History of OpenMI}

The first published OpenMl standard was version 1.4 (Gregersen et al. 2007). It was the result of the European Commission's Framework 5 HarmonIT research project. As well as proving the concept of model integration through a common standard, HarmonIT also prototyped many tools to facilitate making existing models into 'linkable components' and then building and running linked models or 'compositions'. The success of OpenMI 1.4 prompted a second European project, this time part of the LIFE programme, OpenMI-LIFE. OpenMI-LIFE was given a 'best of LIFE programme' award in 2012. The main thrust of OpenMI-LIFE was to demonstrate the OpenMI standard being used in a real-world environment on real-world problems. However, a lot of thought was given to how the development of OpenMI would continue once OpenMI left the research environment and had to stand on its own feet. How could its development be continued? How would its users be supported? How could the outside world be convinced that these mechanisms were in place, working and would be sustained? At that time adoption of the OpenMI represented a significant commitment of resources. To address these points the OpenMI Association (OA) was founded as a legal entity under Dutch law and took ownership of the IPR of OpenMI. To demonstrate that it was a functioning organisation, its first task within the LIFE project was to produce version 2.0 of OpenMI.

As that moved towards completion, the Open Geospatial Consortium approached the OA, explained its growing awareness of the importance of the time dimension and of modelling to its work and suggested that the OA and OGC collaborate. The OA was delighted as this would enable it to publish the OpenMI under the banner of an internationally recognised standards organisation and hence reach a world-wide audience spanning almost every discipline and both the public and private sectors. This has now been achieved.

In parallel, a number of influential hydraulic and hydrological institutions and laboratories including Deltares (https://www.deltares.nl/en/), DHI (https://www.dhigroup.com/) and HR Wallingford (http://www.hrwallingford.com/) created implementations of the standard and applied it to their own numerical model inventories. Since its release, OpenMI 2.0 has been downloaded over 13,000 times, with active interest from over 30 countries. Interest has been observed from a wide range of specialisations ranging from internal combustion engines to human biology and the number of implementations increases. 


\section{Overview of OpenMI 2.0}

Standards can only enable creativity and interoperability if they are properly defined and kept as simple as possible. If this is not the case then the overhead of using them can stifle any advantage (see Iwanaga (2018)). Each aspect of the standardisation must be necessary and proportionate. OpenMI therefore incorporates aspects necessary and proportionate for independent numerical models to be integrated.

At its heart, integrated modelling is about converting a question into a workflow and providing a quantified solution. This involves combining data and models such that the solution can be obtained. The model can be thought of as a unit operation (Figure 1) and be seen as having two facets: model codes which represent system processes / behaviour programmatically and model instances which are the application of the model code to a particular area or problem. By considering a model as a unit operation and defining the input and output data such that the output from one model code can be passed to another, then a linked series of models and an associated workflow can be created. OpenMI uses this paradigm by observing that a composition of models can be thought of as a unit operation made up of independent components.

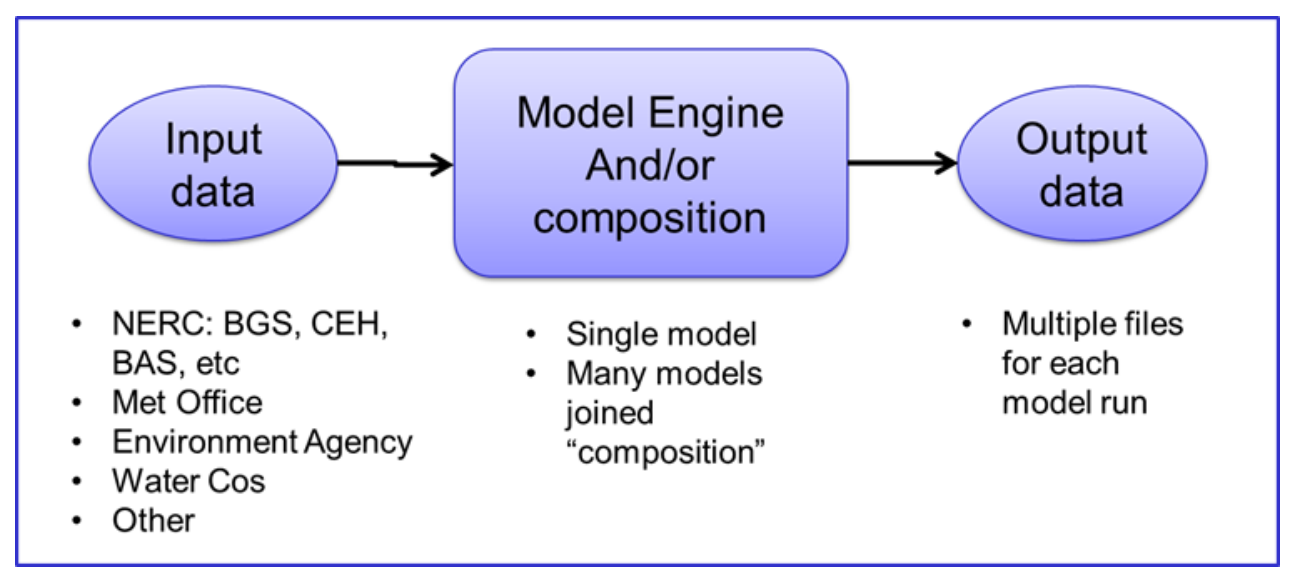

Figure 1: Model as a "unit operation"

OpenMI 2.0 allows those writing numerical models to adapt them so that they can be combined into compositions containing other models that have been similarly modified. In technical terms OpenMI is a set of object interfaces that will form a wrapper around each numerical model code. It can be implemented in any object oriented programming language. When made OpenMI 2.0 compliant, a numerical model becomes a 'Linkable Component' which can then be run in compositions including other Linkable Components. Some of the key ideas and principles implemented by OpenMI 2.0 in forming Linkable Components are given in the sub-sections following.

\section{Control and Structure}

The OpenMI 2.0 interfaces act as a code wrapper around the core numerical model code. Control of the Linkable Component's ability to perform typical functions such as Initialise, Run, Finalise (IRF) are offered to an external, unspecified entity. Usually, numerical models are supplied with an interface, often graphical (a GUI) to enable the user to set parameter values (such as the location of input datasets), set the model running and monitor its progress. However, if the model is to run in an OpenMI 2.0 composition, even if an instance of it has to be created using a GUI, it must be possible for an external entity to control the process of executing the model code.

Achieving this requires that the model code has a certain characterisation: 
- Parameters for the model to run, which are not being offered to other components - such as the location of input or output files - must be independently accessible once the model is set up to run.

- The 'initialise' and 'finalise' functions need to be distinct and control of them available to the external entity which will be running the code.

- The core model function (often consisting of calculating values at a sequence of intervals in time) must also be distinct and control of it available to the external entity.

Indeed, if an OpenMI-compliant component is to run in one of the existing GUIs then it shall be associated with an XML file (called an 'OMl' file) which can be successfully validated against the LinkableComponent.xsd schema (Annex B, OGC OpenMI, 2014).

\section{Exchange Items}

Numerical models will produce a set of results as a set of values for a defined phenomenon or parameter, for example air temperature, water depth, current direction or shoreline position. In some cases, this can be a very long list. As part of making a numerical model OpenMI compliant, the model developer chooses which of their output variables - belonging to the Linkable Component to make available to other numerical models. Each one of these becomes an 'Output Exchange Item', see IBaseExchangeltem and IBaseOutput (OGC OpenMI, 2014, section 6.9).

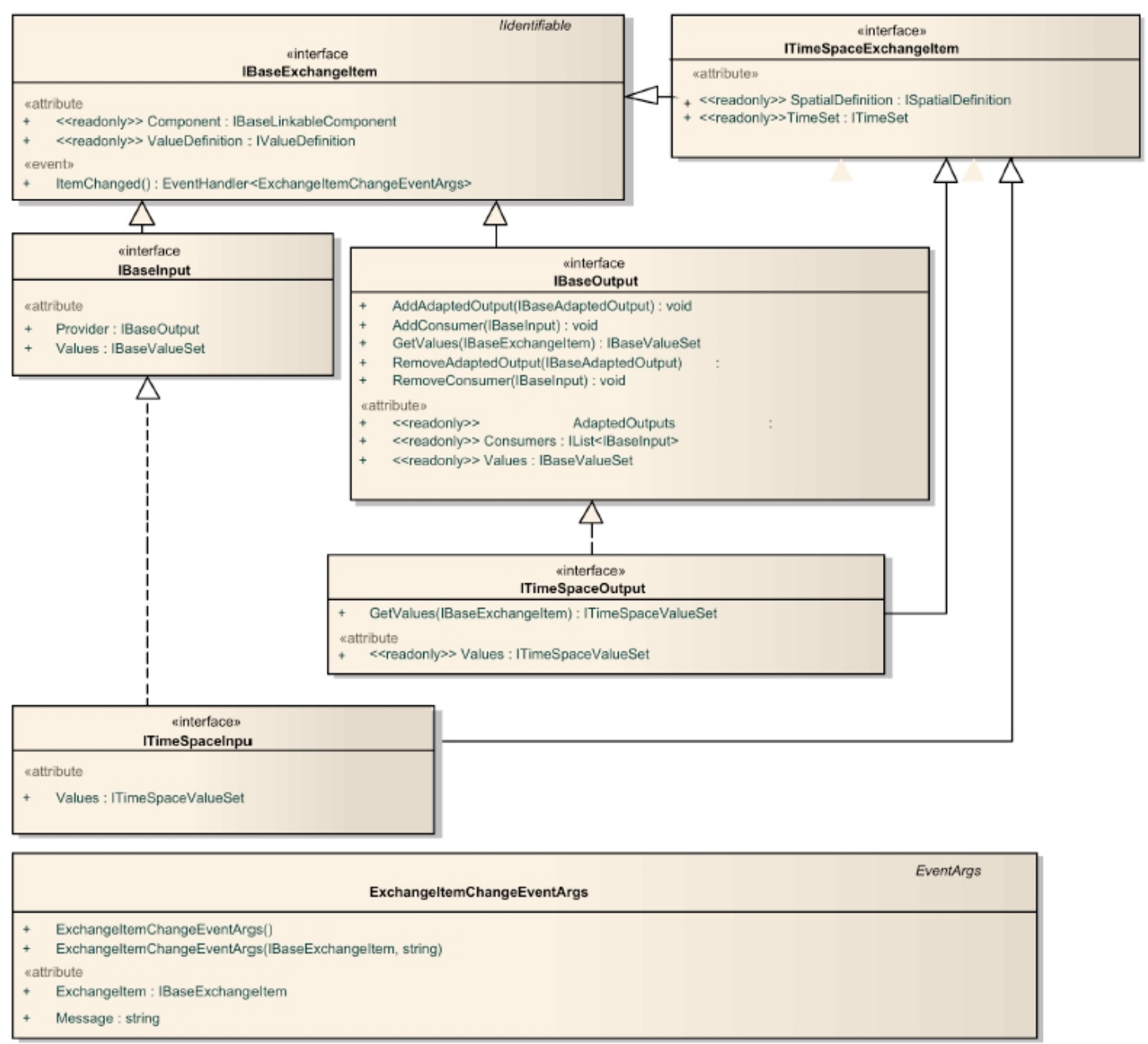

Figure 2: UML Class Diagram for IBaseExchangeltem 
Similarly, numerical models are able to - and usually require - phenomena or parameter values from external sources, for example a simulation of near shore waves will require a bathymetry dataset describing the sea bed. This phenomenon may be variable in time and it may be possible for a numerical model to receive this data from a second numerical model. When a model is made OpenMI compliant, such inputs are designated 'Input Exchange Items', see IBaseExchangeltem and IBaselnput (OGC OpenMI, 2014, section 6.9).

Only Exchange Items will be able to be passed from one numerical model to another via the OpenMI interface.

\section{Parameters and Units}

If two model components are exchanging and using values associated with a phenomenon across an Exchange Item, then the phenomenon itself and its associated units must be clearly and mutually defined. We cannot have a situation where one numerical model is passing 'Wave Height' to another model where the former understands it to be 'Significant Wave Height', measured in metres and the latter understands it to be 'Maximum Wave Height', measured in feet. Thus, OpenMI breaks down the phenomena passed between the exchange items into its DimensionBase as part of the IValueDefinition interface (Length in metres, Mass in kilograms, Time in seconds, ElectricCurrent in amperes, Temperature in kelvin, AmountOfSubstance in moles, LuminousIntensity in candela, Currency with no base quantity) (Table 3, OGC OpenMI, 2014). The IQuality interface inherits from the IValueDefinition interface to allow non qualitative values (e.g. high, low) to be passed, for example, as strings. In the case where the units are dimensionless, represent logarithmic scales or are otherwise problematic when expressed in $\mathrm{SI}$, then extra attention should be paid to the descriptive part of the unit to clarify understanding of the meaning of its value.

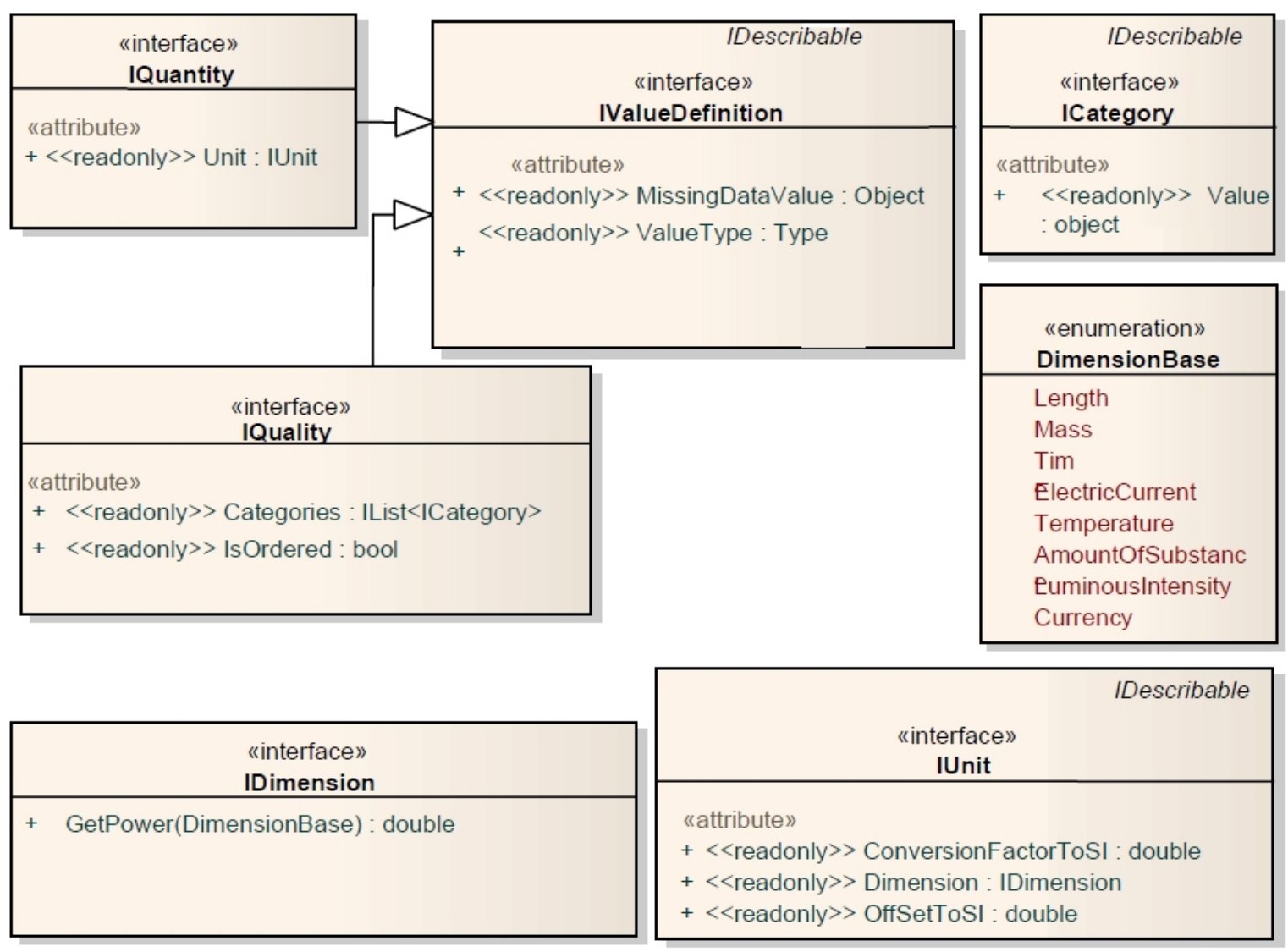


Figure 3: UML Class Diagram for IValueDefinition

This, nor indeed any, definition can be perfect and completely prevent errors in definition or unit, but this structure is intended to offer enough rigour to maximise the opportunity to trap and correct such inconsistencies.

\section{Spatio-Temporal Structure}

Numerical models typically produce their results according to a specified (or assumed) spatiotemporal structure. Harpham (2018) defines a vocabulary for describing these structures as given in Table 1. Such vocabularies constitute important information which can be referenced in metadata associated with models (Harpham and Danovaro, 2015).

\begin{tabular}{|l|l|l|l|l|l|l|}
\hline $\begin{array}{l}\text { Spatial } \\
\text { Structure }\end{array}$ & Point & PointSet & Polyline & PolylineSet & Polygon & $\begin{array}{l}\text { PolygonSet } \\
\text { (Grid, Mesh) }\end{array}$ \\
\hline $\begin{array}{l}\text { Temporal } \\
\text { Variation }\end{array}$ & $\begin{array}{l}\text { Point } \\
\text { Series }\end{array}$ & PointSet Series & $\begin{array}{l}\text { Polyline } \\
\text { Series }\end{array}$ & $\begin{array}{l}\text { PolylineSet } \\
\text { Series }\end{array}$ & $\begin{array}{l}\text { Polygon } \\
\text { Series }\end{array}$ & $\begin{array}{l}\text { PolygonSetSeries } \\
\text { (GridSeries, } \\
\text { MeshSeries) }\end{array}$ \\
\hline $\begin{array}{l}\text { Temporal } \\
\text { and } \\
\text { Spatial } \\
\text { Variation }\end{array}$ & Point & $\begin{array}{l}\text { PointSetTrack / } \\
\text { Developing } \\
\text { PointSet }\end{array}$ & $\begin{array}{l}\text { Polyline } \\
\text { Track }\end{array}$ & $\begin{array}{l}\text { PolylineSet } \\
\text { Track/ } \\
\text { Developing } \\
\text { PolylineSet }\end{array}$ & $\begin{array}{l}\text { Polygon } \\
\text { Track }\end{array}$ & $\begin{array}{l}\text { PolygonSetTrack } \\
\text { / Developing } \\
\text { PolygonSet } \\
\text { (Adaptive Grid, } \\
\text { Adaptive Mesh) }\end{array}$ \\
\hline
\end{tabular}

Table 1: Controlled Vocabulary for Spatio-Temporal Data Structures

The most common of these are well understood and established such as:

- PointSeries, describing a time series of values associated with a single, unmoving point in space, such as readings from a rain gauge;

- GridSeries (given as a special case of a PolygonSetSeries), describing timeseries output associated with points arranged in a rectangular grid with one value for each point for each timestep;

- PointTrack representing a point moving with time, such as the position of a moving vessel.

However, more complex spatio-temporal structures are produced by some numerical models. For example, a probabilistic shoreline erosion model would produce output at a series of timesteps. Output at each timestep is a set of polylines describing the possible position of the shoreline at different rates of erosion. Thus the output across all timesteps could be described as a 'PolylineSetTrack' or 'DevelopingPolylineSet', as illustrated in Figure 4. 


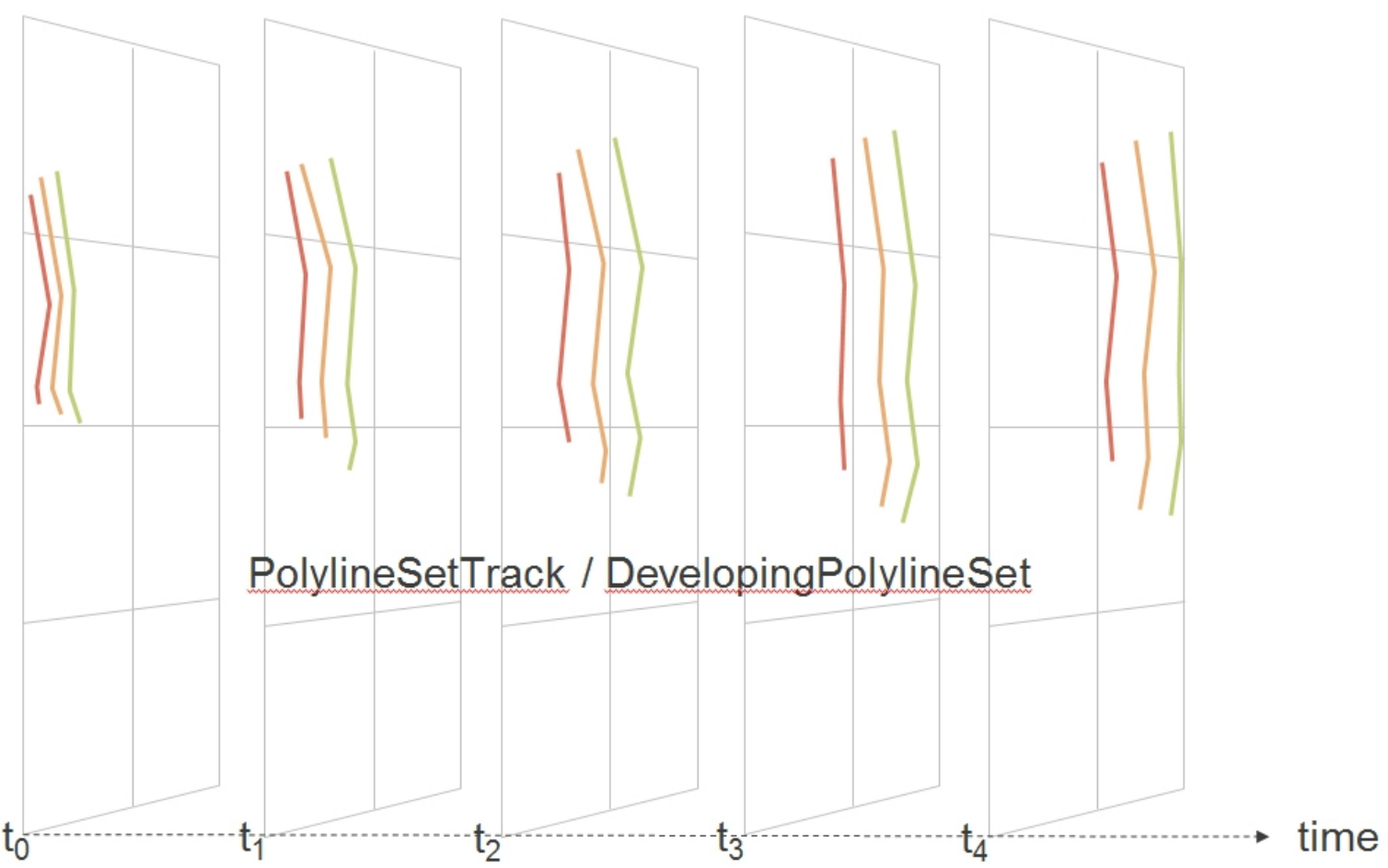

Figure 4: PolylineSetTrack - Probabilistic Progressive Shoreline Position

OpenMI is designed to maximise flexibility and so, to incorporate all common (and less common) numerical model outputs in one, two and three dimensions, the spatial and temporal components have been separated. Indeed, the core OpenMI standard does not assume that the numerical model will have a timestepping aspect at all; this is offered as one of the optional extensions to the standard - albeit a commonly used extension.

OpenMI 2.0 breaks down the spatial aspect of numerical model outputs into its basic spatial constructs. This is governed by the 'ISpatialDefinition' interface (OGC OpenMI, 2014, section 6.4) including an 'ElementCount', 'SpatialReferenceSystem' and 'Version'. The 'Element Set' of the spatial structure 'IElementSet' includes management of indexes, counts and coordinates, with each element enumerated into five types: IdBased, Point, Polyline, Polygon and Polyhedron.

Where required, time series values for the Element Set are described by the ITimeSpaceValueSet interface (OGC OpenMI, 2014, section 6.6). Also included and of particular importance for running time series compositions of numerical models is the TimeHorizon property of the ITimeSet interface (OGC OpenMI, 2014, section 6.5), which describes the full time interval governed by the numerical model. 


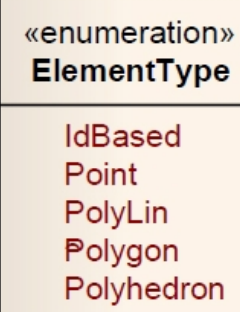

Figure 5: UML Class Diagram for ISpatialDefinition

\section{Adaptors}

Two independently developed OpenMI 2.0 compatible numerical models can have their inputs and outputs connected according to the desire of the modeller: input exchange items to output exchange items. However, this is unlikely to be feasible without adjustment. For example, the first model may produce its results as a rectangular GridSeries and the second model may wish to receive them as a PolylineSeries; the first model may produce its results in metres and the second model may wish to receive them in feet; the two models may use a different vertical datum. Therefore, to be connected correctly an 'adaptor' will be required across the connection between input and output exchange items in order to perform the necessary adjustments, see Figure 6 where the notation used is that adopted by Harpham and Hummel. Such adaptors are created using the interface IAdaptedOutputFactory and managed by the interface IBaseAdaptedOutput (OGC OpenMI, 2014, section 6.10). 
Figure 6: Use of an adaptor to handle differences between input and output exchange items, Harpham / Hummel notation.

It is possible to nest multiple adaptors and apply them in turn and in combination. For example, one adaptor may perform a unit conversion required between two models and another may perform a datum conversion additionally required by a third model. This situation is illustrated in Figure 7 using the notation adopted by Harper. It seems that only individuals with family names beginning with the letter ' $\mathrm{H}$ ' are able to create notation for adaptors.

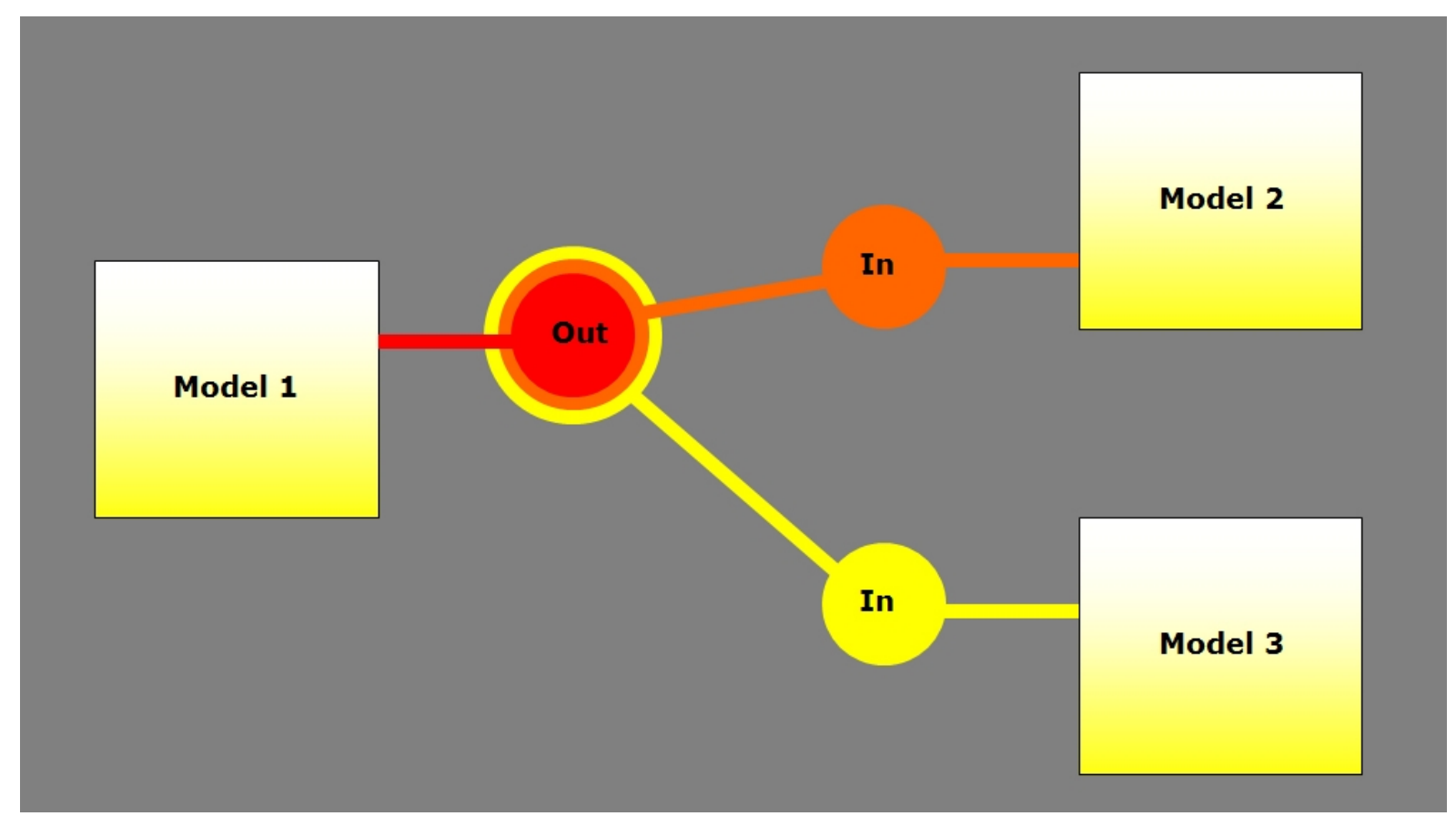

Figure 7: Nested adaptors shown using the Harper notation.

\section{Changes from OpenMI 1.4:}

There are a number of key differences from OpenMI 1.4. In addition to some lower level technical changes, the main aspects are as follows: 
- Base Interfaces and Extensions - A set of minimum 'base interfaces' for compliance, plus the addition of extensions (including an extension covering time and space dependent components). In particular, the essential OpenMl component is no longer forced to be time and space dependent, making the standard considerably more flexible and extensible. This allows different types of components to be incorporated e.g. those which vary in time and not in space; those which vary in space, but not in time or those which vary in both time and space.

- Adaptors - Taking over from the role of 'Data Operations' in OpenMI 1.4, 'Adapted Outputs' allow multiple, distinct adaptations, separate from the components themselves and the link, to take place. Again, this makes the standard more flexible and allows outputs and adapted outputs to be re-used by more than one OpenMl component.

- Events - OpenMI 1.4 had its own in-built event system. For version 2.0 the standard .NET and Java event mechanisms are utilised.

- Data Definitions - Values passed in OpenMI 1.4 could only be quantitative. OpenMI 2.0 supports qualitative information (e.g. text values such as 'high' and 'low'). It also incorporates an extended element set to follow the OpenGIS standard with no distinction between $2 \mathrm{~d}$ and $3 \mathrm{~d}$ spatial structures, improved handling of timestamps and timespans, and a more flexible value set to include objects as well as scalars and vectors.

\section{Making new and existing models OpenMI 2.0 compliant}

The original vision and purpose behind OpenMl was to create a method for easy integration of both new and existing numerical models - to build on the existing investment in the development of numerical models. The method provided is to make each numerical model 'OpenMI 2.0 Compliant', that is, make each numerical model code into an OpenMI 2.0 Linkable Component, adhering to the standard. Compositions of multiple components can then be put together and run as a collective with data exchange between linked components in the composition.

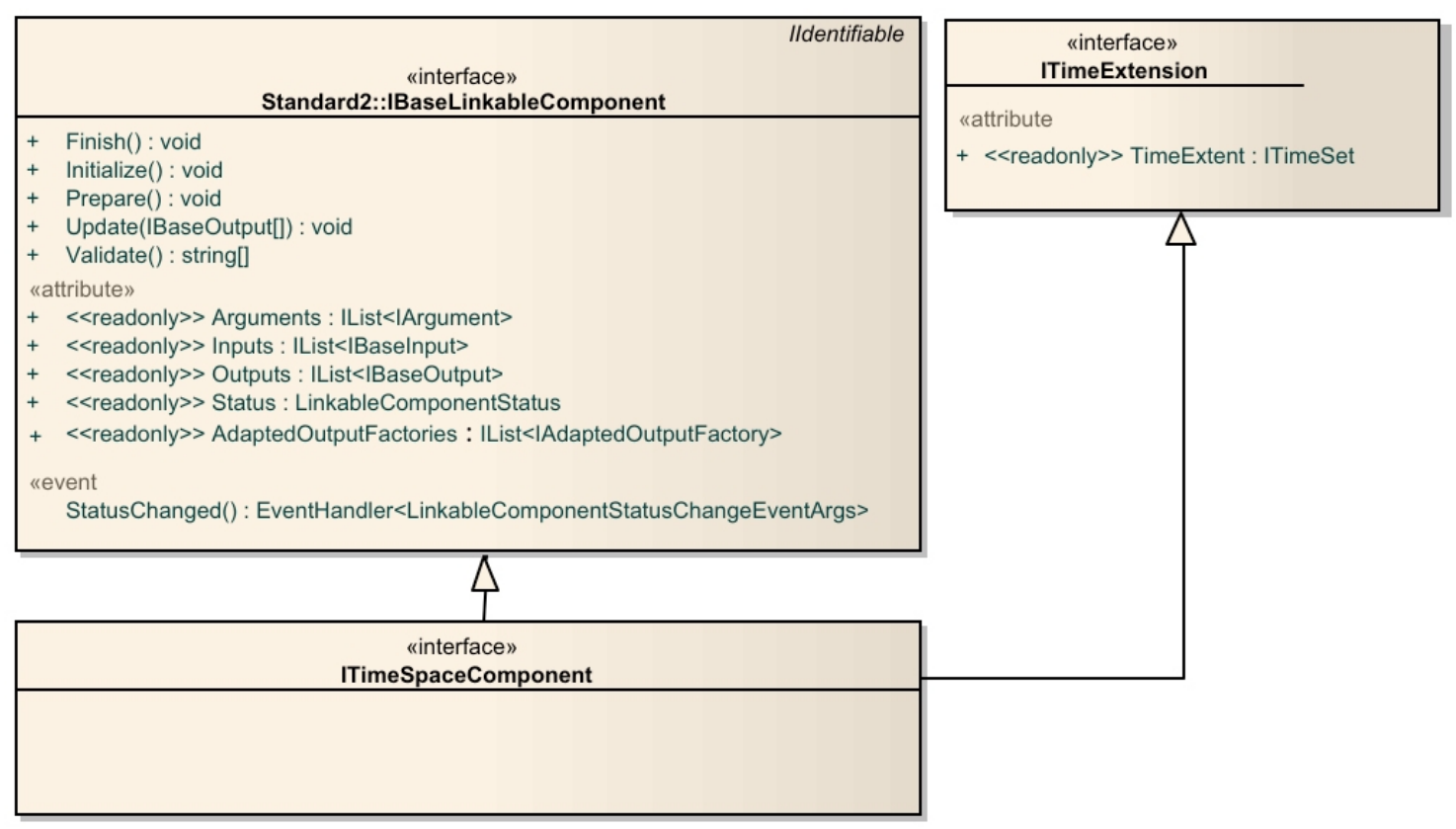

Figure 8: UML Class Diagram for IBaseLinkableComponent 
A numerical model (or equivalent) is OpenMI 2.0 compliant if it implements a set of interfaces that can connect to and interact with the OpenMI 2.0 component interface IBaseLinkableComponent or its specialisations (such as the ITimeSpaceComponent). These interfaces are described in Clause 6, 'OpenMI Requirements Classes' of OGC OpenMI (2014). In particular, requirements for compliance can be summarised as follows:

1. An OpenMI 2.0 compliant component shall implement the mandatory core requirements given in clause 6 of OGC OpenMI (2014).

2. Two further optional core interfaces (IManageState and IByteStateConverter) may be implemented. If implemented then this must be done according to the OGC OpenMI (2014) specification.

3. Optional OpenMI 2.0 extensions are also given. If implemented, this must be done according to the OGC OpenMI (2014) specification.

4. An OpenMI 2.0 Compliant component (including its extensions) shall, when compiled, reference the OpenMI.Standard2* .Dlls (.Net framework 2.0 or higher) or OpenMIstandard2*.jars (java 1.5 or higher) as released by the OpenMI Association (OpenMI Source Forge).

Thus, making a numerical model OpenMI 2.0 compliant can be achieved in two steps:

- Step 1: Prepare the numerical model code to be consistent with the principles outlined in the above and for mapping to the OpenMI 2.0 interfaces i.e. with respect to Control and Structure, tidy and accessible arrangement of input and outputs to be accessed through Exchange Items, clear definitions of Parameters and Units, clear definition of SpatioTemporal Structures, and understanding of any potential requirements for use of Adaptors.

- Step 2: Connect the relevant attributes of the numerical model into the OpenMI 2.0 interfaces i.e. 'wrap' the model. Software Development Kits (SDKs) are provided with implementations of OpenMI to aid developers in this task (Harper et al., 2016; Harpham et al., 2014).

If the numerical model is being written from scratch then the principles required by Step 1 can be employed directly and the model written according to the OpenMI 2.0 standard; if the numerical model already exists then these principles will need to be retrofitted.

\section{Creating and Running OpenMI 2.0 Compositions}

OpenMI 2.0 components can be run individually, as single numerical models, taking input from the set of supporting data files as standard for the model or they can be run as compositions of multiple components which are able to pass data between them as they run, across Exchange Items. It is the responsibility of the modeller to ensure that any connections are scientifically valid. Although OpenMI includes low-level definitions of each aspect of this connection (e.g. spatial structure, parameter), it can provide no specific validation. Indeed, other more subtle aspects, such as how the data is represented within each spatial element, must also be considered when connections are constructed. For example, if the spatial element is a polygon - say a grid cell - then is the value taken by the cell attributed to its centroid or its corners? If the corners all have different values, then which of these is passed to another model which has a single point linked to the grid cell?

When an OpenMI component (numerical model) takes input from another component then this overrides the associated input it would otherwise get from its supporting data (configuration or input data file), as illustrated in Figure 9. The Hydrological Drainage Model would usually be set up taking driving data from a rainfall time series, perhaps from an in-situ instrument. This input can be 
validly replaced by input from another OpenMI 2.0 component which is a numerical model producing rainfall time series output. Thus, the rainfall Input Exchange Item from the Hydrological Drainage Model is connected to the rainfall Output Exchange Item from the Rainfall Model. The Hydrological Drainage Model will then produce its output in its usual way.
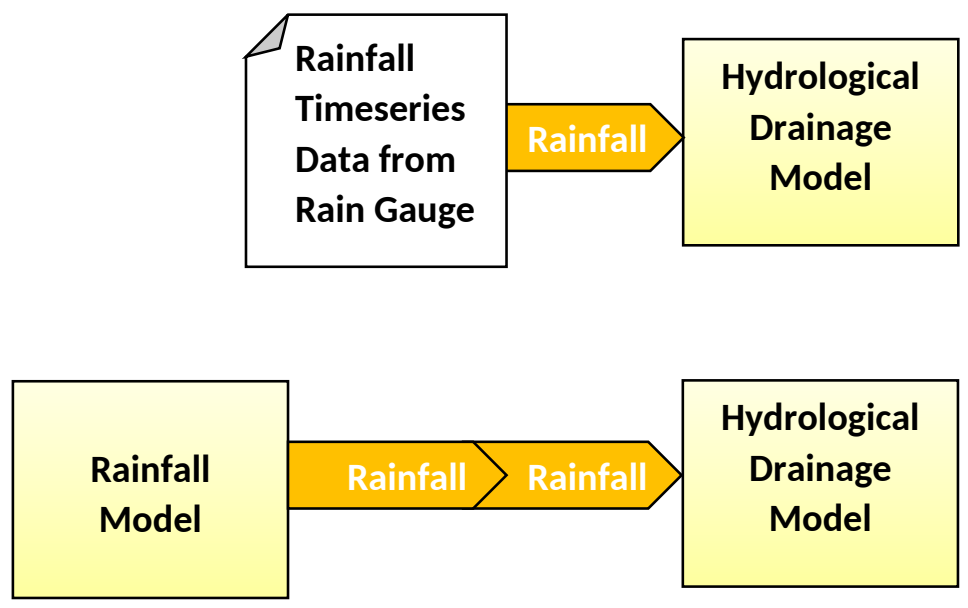

Figure 9: Replacing file-based inputs with a connection across Exchange Items.

\section{Implementations of OpenMI 2.0}

A number of implementations of OpenMI 2.0 are available. These usually consist of i) a Software Development Kit (SDK) to facilitate the wrapping of numerical models into OpenMI 2.0 components and ii) a Graphical User Interface (GUI) to assemble compositions of components into compositions and to run these compositions. The GUI acts as the unspecified external entity which controls the composition and the components within it.

HR Wallingford's FluidEarth (Harpham et al., 2014; Harper et al., 2016) is a typical implementation of OpenMI 2.0. It is provided with the FluidEarth SDK and a GUI called Pipistrelle, with the open source code available at https://sourceforge.net/projects/fluidearth/. Since the OpenMI 2.0 standard is flexible, each implementer has the freedom to apply it according to their particular needs. The FluidEarth implementation uses two sets of configuration files so that each OpenMI 2.0 component is accompanied by two files, a .omi file and a .xml file which offer configurable parameters and a means of engagement with the user interface, Pipistrelle. One of the components, usually one with a time horizon starting earlier and finishing later than all the others, is connected to a 'Trigger'. This component is used to start the composition running and will make requests from other components in the composition in an attempt to pull data from them to satisfy its own requirements. In turn, components will make requests from each other as the composition progresses until it is able to complete. This process is demonstrated by a simple composition involving two ponds (pondWest and pondEast) where pondWest is slowly draining into pondEast. The composition progresses through time steps with pondEast pulling data from pondWest. The sequence diagram for the main functions is shown in Figure 10. 


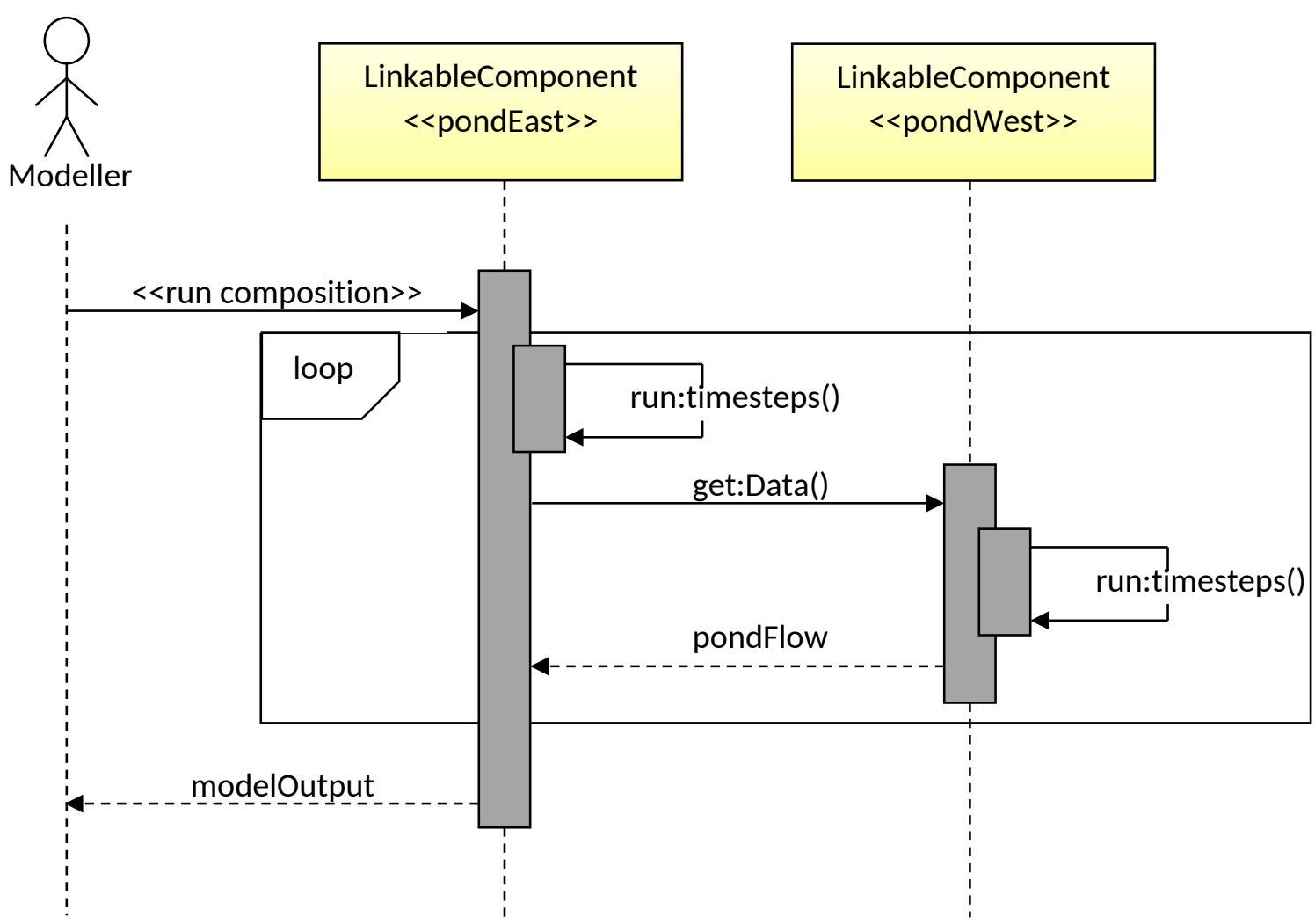

Figure 10: Sequence diagram for run and timestepping elements of simple pond composition

Figure 11 shows this composition in the Pipistrelle GUI where pondEast is attached to the trigger, denoted by the small OpenMI logo. If there are any adaptors present across the link between the two pond components, then this is shown by the bold connecting arrow. Clicking on this arrow will show the attributes of the adaptor(s). 


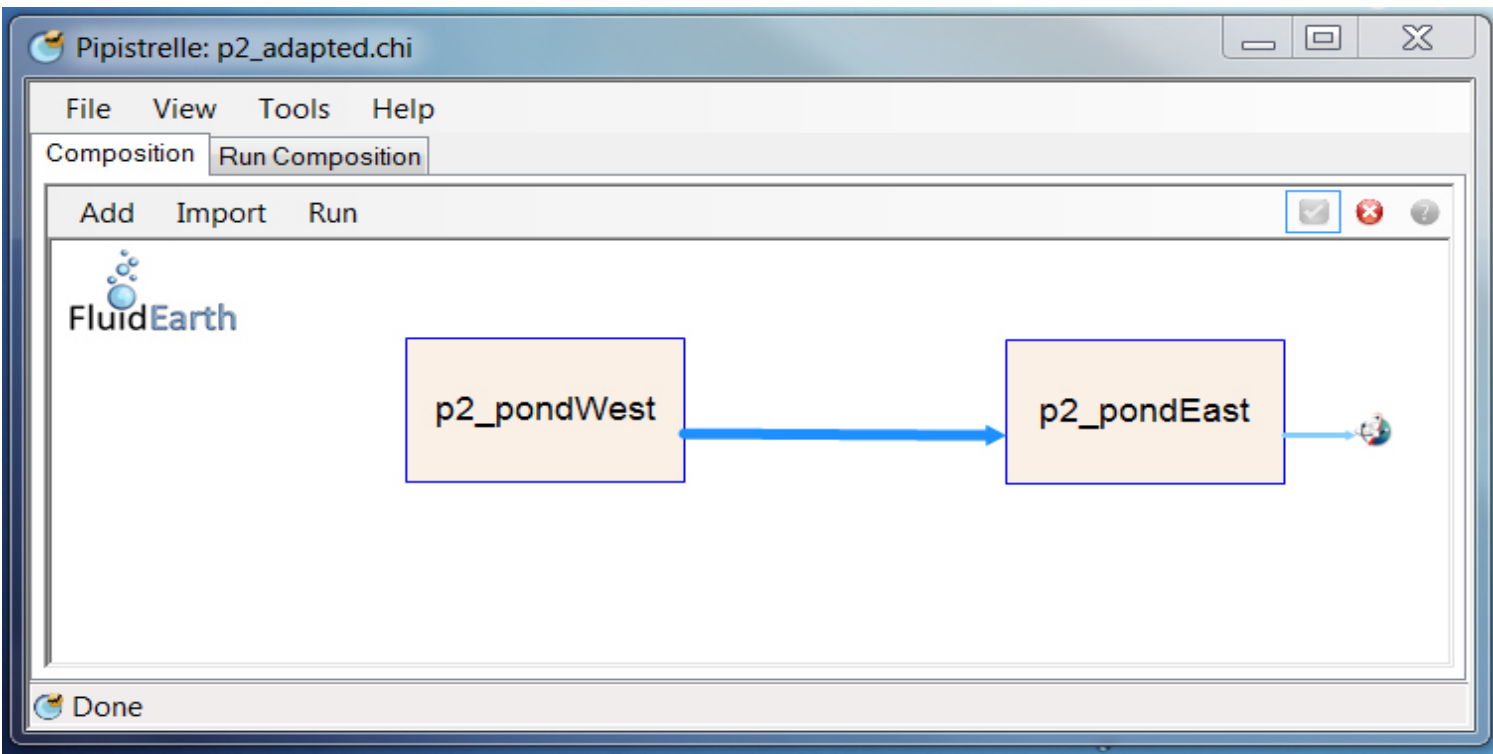

Figure 11: The simple pond composition shown in the Pipistrelle GUI

Pipistrelle will also produce a run log, shown in Figure 12. The run log does not give the model outputs, which are produced in additional files, it merely reports the status of the components and the composition itself.

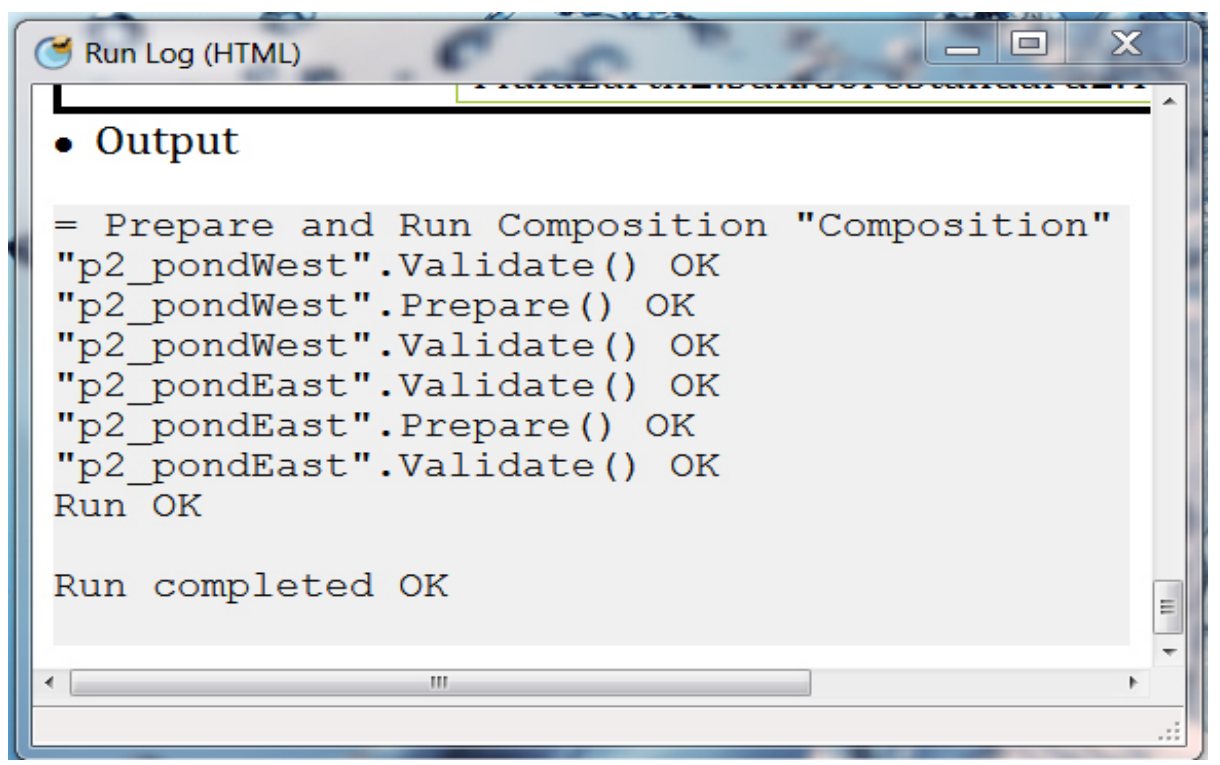

Figure 12: Pipistrelle GUI run log for the simple pond composition

\section{Examples of OpenMI 2.0 Compositions}

River channel - floodplain interactions for flash flooding in Genoa

Harpham et al. (2016) includes an OpenMl composition modelling a flash flood which occurred in Genoa in October 2014. An almost identical event had occurred three years earlier in November 2011 when a third of the average annual rainfall fell in six hours. Both events included one-or-more fatalities and a vast amount of damage was caused. An OpenMI 2.0 composition was used by HR Wallingford to model the downstream hydraulics which studied the interaction between the river channel and the flood plain. Along the river side, flood water passed from the river into the town and also from the streets of the town back into the river. Thus, a two-way connection was required 
between the numerical model simulating the flow in the channel overtopping to the streets and also the numerical model simulating the movement of water through the streets and then back into the channel.

The composition was put together as part of the Distributed Computing Infrastructure for HydroMeteorology (DRIHM) project (D'Agostino et al. 2014, Danovaro et al., 2014, D'Agostino et al., 2015). The FluidEarth implementation of OpenMI 2.0 was used to build the OpenMI components and then assemble and run the composition, which can be seen in Figure 13.

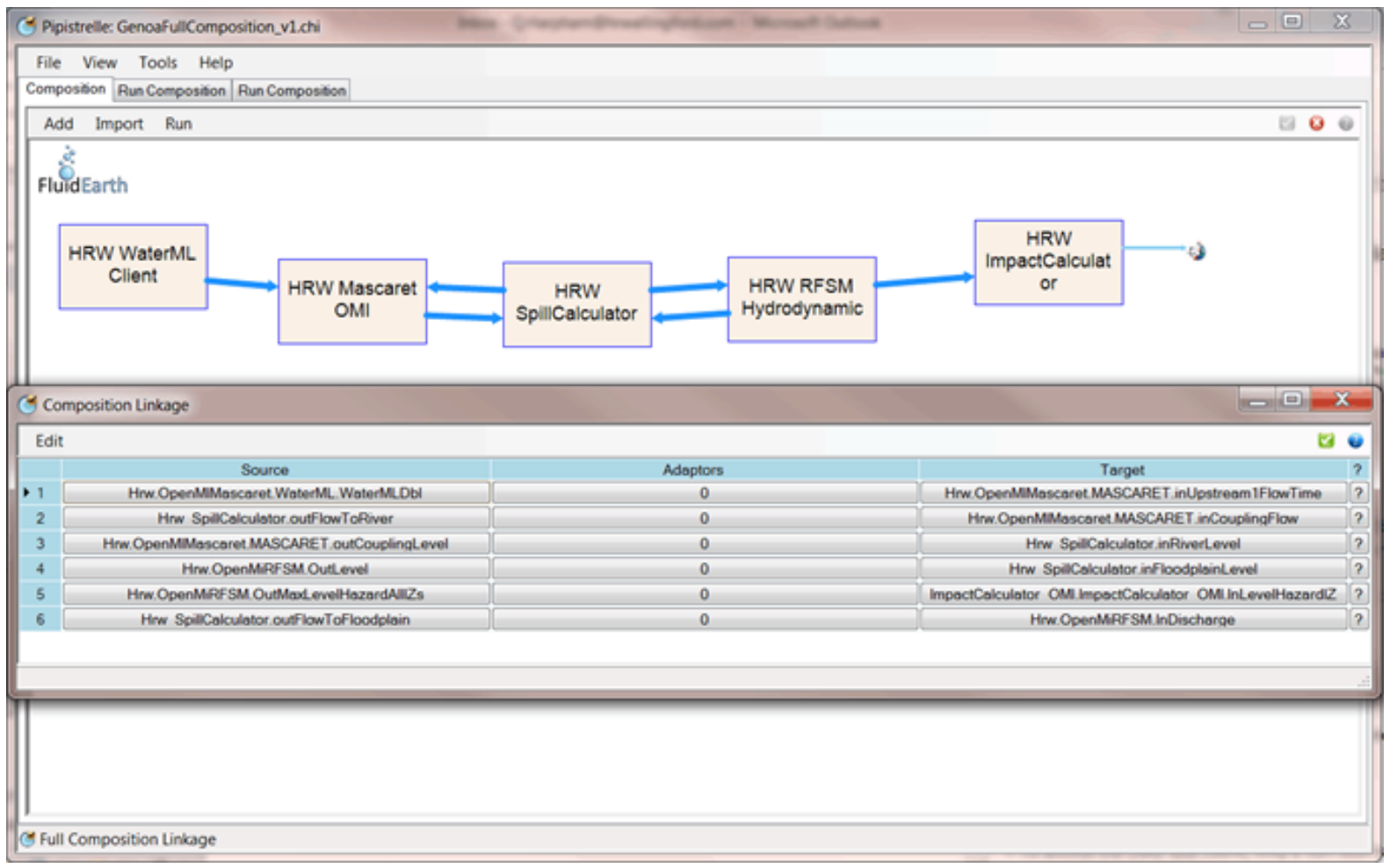

Figure 13: The Pipistrelle GUI showing the OpenMI composition studying the Genoa flash floods of November 2011 and October 2014

The Linkable Components used in the composition are as follows (more detail is given in Harpham et al. 2016):

- HRW WaterML Client: A data ingestion translating the upstream flow-time boundary condition from the WaterML2 standard into that required by the following OpenMI component, in a one-way connection.

- MASCARET (Goutal et al. 2012): simulating the flow in the river channel.

- HRW Spill Calculator: Converting water level to and from flow. This component could also be an adaptor between the MASCARET and RFSM components.

- HRW RFSM Hydrodynamic: A flood spreading model calculating flow across the flood plain, in this case, the streets of Genoa (Jamieson et al. 2012).

- HRW Impact Calculator: a tool used to estimate the impact of flooding on buildings and agricultural land and predicted Loss of Life using the Risk To People method published in the DEFRA report FD2317 (Ramsbottom et al., 2003).

Use of the OpenMI 2.0 composition enabled the flood event to be accurately simulated with water flowing to and from the flood plain. Existing, well established numerical models were able to be linked together into a composition thereby providing a new capability in simulating such events. As 
the water flows through the streets the damage to buildings and hazard to people is calculated by the Impact Calculator Linkable Component. A single frame of the results animation is given in Figure 14 , showing the flow of water through the city as the event progressed. There was a large exchange of water between the channel and the flood plain in the area highlighted as the channel proceeds under the railway line. A high hazard was identified at this point, which is, sadly, where one of the fatalities occurred. The total damage for the 2014 flood was calculated to be $€ 205$ million (at 2014 prices). This was within $2.5 \%$ of the unofficial locally calculated estimates.

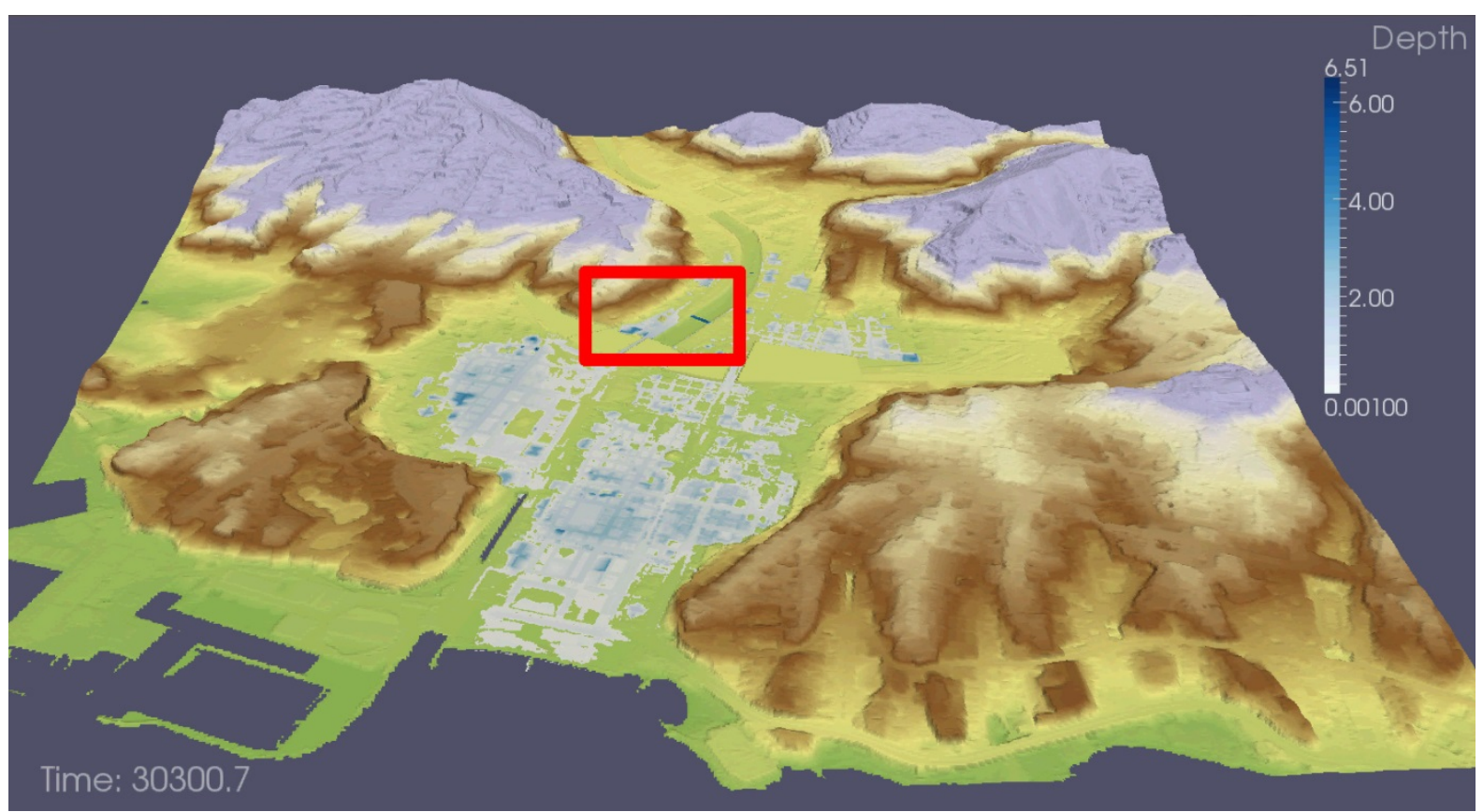

Figure 14: Frame of animation of water flow through Genoa during the flash flood of October 2014

\section{Thames integrated model}

BGS's Thames integrated model (TIM) links the detailed geology of the Thames catchment with groundwater and surface water hydrology including rainfall, runoff, recharge, and to a limited extent the source and resource management applied in the Thames catchment. There has been particular emphasis on the geology of the greater London area (Royse et al., 2012). The project brings together a unique combination of geological, hydrogeological, environmental and socio-economic challenges that are intrinsically linked and impacted by climate change. To address these challenges requires fully attributed 3D models that incorporate information and processes from all of these disciplines so that accurate representations, simulations, forecasts and predictions can be made. These forecasts and predictions are required to enable informed decision making and planning for sustainability. In particular, integrated modelling is being applied to understand the interaction between rainfall evaporation and runoff, together with river flows for the Thames and its tributaries, with groundwater flow.

The importance of interaction between the surface water system and abstraction has been demonstrated by using the TIM linked model composition developed by Mansour et al. (2013). A model composition which allows an appropriate representation of the hydrological system has been developed and is shown diagrammatically in Figure 15. Two groundwater models have been developed: one of the chalk using ZOOMQ3D (Jackson and Spink, 2004) and another of the Jurassic limestones using BGSGW (Mansour et al., 2013). The former is a distributed groundwater flow model and the latter is a semi-distributed lumped parameter model that can be used to simulate 
groundwater behaviour. The boundaries of both models are shown on Figure 16 and it should be noted that for the chalk model these extend outside of the Thames Basin. This is to ensure that sensible groundwater flow boundaries are defined so as not to erroneously calculate baseflow to rivers whose surface catchments are different to groundwater catchments and unduly affect the impacts of groundwater abstractions.

The two groundwater models are linked via a river model (MCRouter) developed using the Muskingham-Cunge approach (Chadwick and Morfett, 1986). A simple hydraulic river model was chosen to ensure the composition can be tested before including a more complex river model. These three models are driven by run-off and recharge generated by the recharge model ZOODRM (Mansour and Hughes, 2004; Hughes et al., 2008). The run-off is routed to the river model whilst the recharge is passed to the two groundwater flow models (Figure 15). As described above, the Jurassic limestones and chalk aquifer are not linked via the sub-surface, only via interaction with the River Thames and its tributaries. The two groundwater models and the river model are, therefore, dynamically linked by the model linkage standard OpenMI (Gregersen et al., 2007). This linkage has been facilitated by using the Fluid Earth software development kit (SDK) and the associated editor, Pipistrelle, to construct compositions (Harpham et al., 2014).

The model composition has been applied to a hypothetical situation to test the applicability of the models and the benefit is accrued from linking them. A hypothetical groundwater abstraction of 150 Mlday $^{-1}$ has been used to investigate the impacts of groundwater abstraction on the flows in the River Thames. The groundwater abstraction was simulated from a point on the banks of the River Thames (labelled BH on Figure 16). This abstraction was linked to river flow as measured at a downstream gauging station (labelled GS-B on Figure 16). To investigate the effect of different management regimes two scenarios were simulated: One with a fixed groundwater abstraction of $150 \mathrm{Ml} / \mathrm{d}$ and the other with an abstraction that varies from 50 to $150 \mathrm{Ml} / \mathrm{d}$ depending on river flow at GS-B (Figure 16). The relationship between river flow and magnitude of groundwater withdrawal was achieved by the inclusion of an abstraction management component in the composition which modified groundwater abstraction during runtime. This component related groundwater abstraction by a simple rule and was included in the composition. The abstracted water is returned to the river some $35 \mathrm{~km}$ upstream (labelled GS-A on Figure 16) of the groundwater abstraction as this represents typical water use, from abstraction, by the city of Oxford and after use discharge to the sewerage system and hence finally sewage effluent returned to the river.

The model composition was simulated for a 30 year period and groundwater heads and the river hydrograph downstream of the groundwater abstractions plotted (see Figure 3 and 4 in Mansour et al., 2013). During conditions of low flow, i.e. during the $1975 / 6$ drought the model simulation resulted in a river flow that was lower for the scenario with fixed abstraction than for the scenario where groundwater abstraction decreased. However, as groundwater abstraction was reduced, groundwater levels surrounding the abstraction were higher. Whilst the variable abstraction reduced the impact on groundwater heads the overall impact on river flows increased due to the reduction in return flows to the River Thames. Whilst this is a hypothetical example, the results are contrary to expectation and the utility of the modelling composition in the development of management policies for droughts and its potential for other scenarios was demonstrated. 


\section{Recharge}

- 2D distributed model

- Inputs: climate (rainfall and PET)

- Outputs: runoff, soil moisture,recharge ET

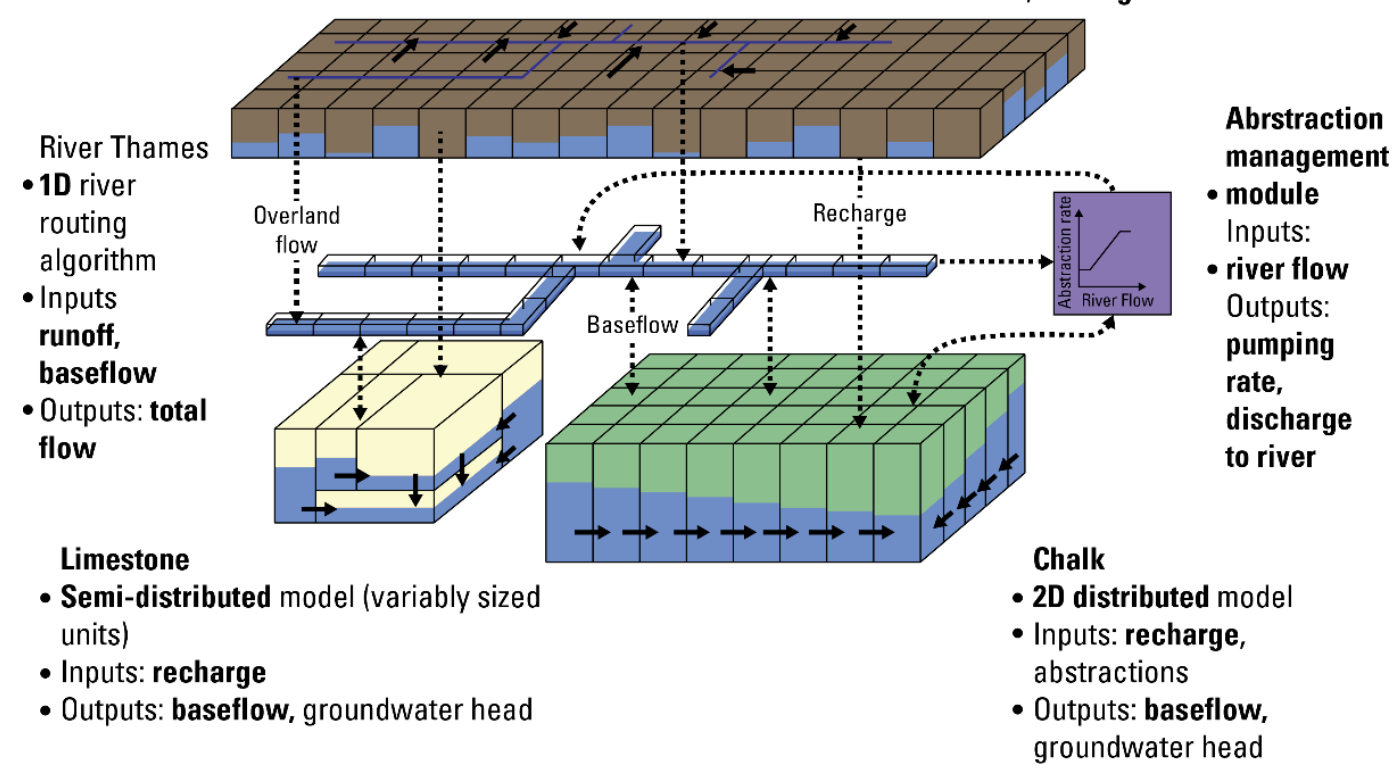

Figure 15: Representation of the hydrological system in the Thames integrated model

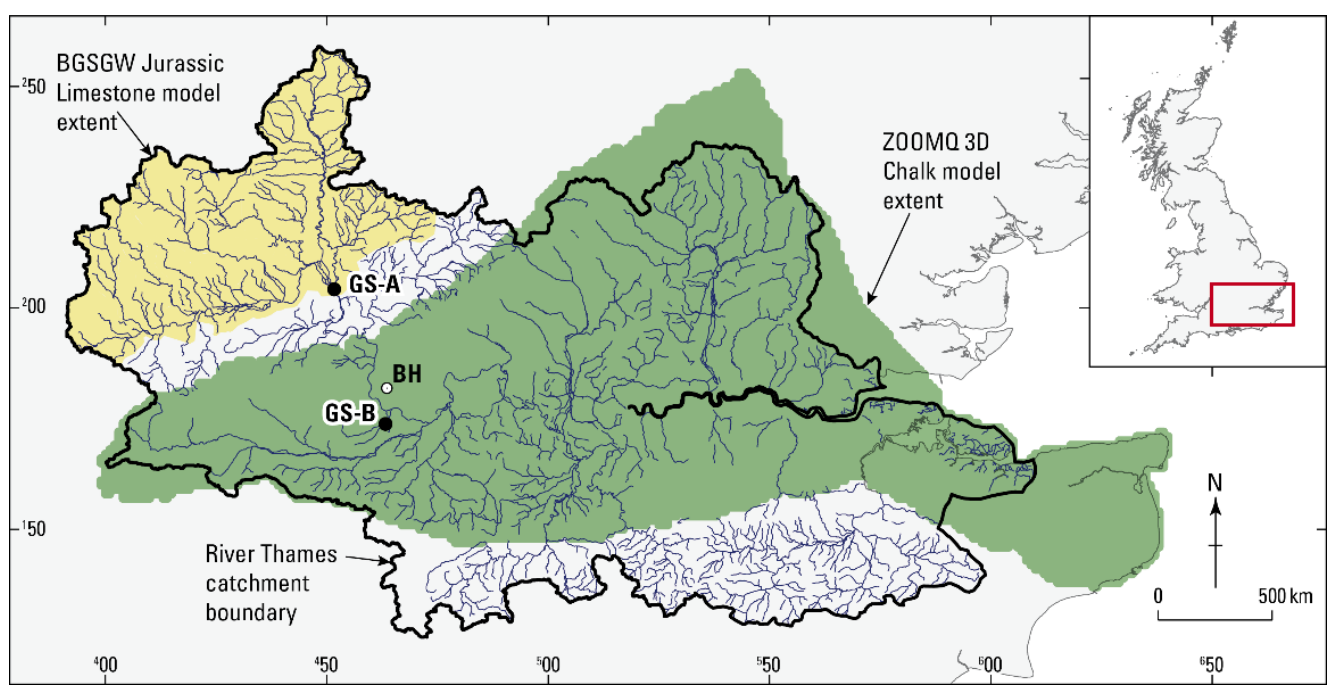

Figure 16: Individual model extents in the Thames integrated model

\section{Conclusions}

Sharing the same purpose and motivation as its predecessor, OpenMI 2.0 has a number of similarities and a number of differences to the earlier version of the standard, OpenMI 1.4. Both have been designed to be as open and flexible as possible and allow numerical models to be coupled easily in a manner which allows two-way passage of data between them whilst they are in the process of running. In this way, the numerical models can influence each other. The basic constructs 
are the same: compliant numerical models become Linkable Components offering and receiving Exchange Items to and from each other. No assumptions are made about the spatial structures involved with both versions of the standard offering low level spatial constructs, rather than assuming that Grids or Meshes will be used. Similarly, parameters are broken down into their Dimension Base offering a low level definition, designed to reduce the probability of errors in the passage of data along two connected Exchange Items.

Unlike version 1.4, OpenMI 2.0 does not assume that the models used will be stepping through time as they run - although this is most often the case - and offers timestepping as an extension to the base standard. In order to offer the modeller maximum flexibility, version 2.0 defines any adaptation of outputs or inputs as a separate entity (an adaptor) to the Linkable Components rather than embedding such an adaptation in one of the Linkable Components. Also, the passage of data between Linkable Components is more efficient in version 2.0 of the standard.

There is a trade-off between flexibility and performance. Coupling numerical models in this highly flexible way can therefore cause performance degradation. The run time of a composition of two connected numerical models will often be more than the sum of the independent run times. This degradation varies depending on the implementation. In the worst cases, this is the sum of the two run times plus the communication time passing data plus the extra run time caused by breaking up the run of each model; at best the composition runs for slightly longer than the longer of the two run times. Although individual numerical models can still benefit from parallel high performance computing (HPC) architectures while running as linkable components, OpenMI 2.0 doesn't include a specific support for running model compositions on HPC. However, such a specification has been explored by Buahin and Horsburgh (2018), offering HydroCouple interface definitions for consideration in future versions of the OpenMI standard.

The integrated modelling solution offered by OpenMI 2.0 has been demonstrated to work successfully and offer numerical modellers a valid method for the two-way coupling of numerical models. In many ways, any alternative solution would have to function similarly: numerical models would need to offer clear outputs and clear inputs; control would need to be passed to an external entity so that a controllable composition is possible; some sort of adaptation would be required across connections to deal with any inherent differences between candidate inputs and outputs. Thus OpenMI 2.0 offers a natural and intuitive method for integrating numerical models.

OpenMI has originated from the domain of hydraulic modelling. It has been devised to integrate such things as models of drainage systems with models of river systems; models of groundwater with models of surface water. However, it can be observed that similar concepts exist in other spheres. A water supply network is a pumped system of pipes under pressure, entirely analogous to a biological bloodstream. An internal combustion engine cylinder also contains a set of separate yet integrated processes as the gases are introduced, ignited and exhausted from a cylinder. As such, OpenMI 2.0 can be used in all circumstances where it is necessary to exchange data between numerical models, in particular when the models must be allowed to influence each other as they proceed.

\section{Acknowledgements}

OpenMI 2.0 and the worked examples given here are the result of over a decade of research by a wide variety of individuals. The projects include:

- HarmonIT (EC contract: EVK1-CT-2001-00090) and OpenMI-Life (Grant agreement number LIFE06 ENV/UK/000409)); 
- DRIHM (EC Grant Agreement 283568) and DRIHM2US (EC Grant Agreement 313122);

- FluidEarth, HR Wallingford.

The authors would also like to acknowledge the technical contribution from the OpenMI Association Technical Committee (OATC) and support throughout the standardisation process from the Open Geospatial Consortium (OGC).

More recent incorporated research into spatio temporal structures has been conducted as part of the UKSA IPP 'D-MOSS' project building and implementing a dengue fever forecasting system for Vietnam (https://www.gov.uk/government/news/new-projects-see-uk-space-firms-tacklesoutheast-asian-challenges).

\section{References}

Anastas, P. 2010 Agency Priority. EPA Office of Research and Development, Washington, D.C.

Buahin, C. and Horsburgh, J. 2018. Advancing the Open Modelling Interface (OpenMI) for Integrated Water Resources Modeling, Environmental Modelling \& Software, 108, pp 133-153. doi 10.1016/j.envsoft.2018.07.015.

Chadwick, A. and Morfett, J., 1986. Hydraulics in Civil Engineering. Allen and Unwin (Publishers) Ltd, London, UK.

D’Agostino, D., Clematis, A., Galizia, A., Quarati, A., Danovaro, E., Roverelli, L.,Zereik, G., Kranzlmuller, D., Schiffers, M., gentschen Felde, N., Straube, C.,Caumont, O., Richard, E., Garrote, L., Harpham, Q., Jagers, B., Dimitrijevic, V., Dekic, L., Parodi, A., Fiori, E. and Delogu, F., 2014 The DRIHM project: a flexible approach to integrate HPC, grid and cloud resources for hydro-meteorological research, SC '14: Proceedings of the International Conference for High Performance Computing, Networking, Storage and Analysis. pp 536-546, IEEE Press Piscataway, NJ, USA ( 2014 . doi 10.1109/SC.2014.49.

D'Agostino, D., Danovaro, E., Clematis, A., Roverelli, L., Zereik, G., Parodi, A. and Galizia, A. 2015. Lessons learned implementing a science gateway for hydrometeorological research. Concurrency and Computation: Practice and Experience.

Danovaro, E., Roverelli, L., Zereik, G., Galizia, A., D'Agostino, D., Quarati, A., Clematis, A., Delogu, F., Fiori, E., Parodi, A., Straube, C., Felde, N., Harpham, Q., Jagers, B., Garrote, L., Dekic, L., Ivkovic, M., Richard, E. and Caumont, O. Setup an hydro-meteo experiment in minutes: the DRIHM einfrastructure for hydrometeorology research. Proceedings e-Science 2014: 10 ${ }^{\text {th }}$ IEEE International Conference on e-Science, Guarujá, SP, Brazil, October 20-24, 2014.

Gregersen, J. B, Gijsbers, P. J. A. and Westen, S. J. A. 2007. OpenMI: Open Modelling Interface, Journal of Hydroinformatics Issue 9(3) 2007 pp 175-191.

Goutal, N., Lacombe, J-M., Zaoui, F. and El-Kadi-Abderrezzak, K. 2012. MASCARET: a 1-D OpenSource Software for Flow Hydrodynamic and Water Quality in Open Channel Networks, River Flow 2012 - Murillo (Ed.), pp. 1169-1174.

Harper, A., Barnes, J. Cleverley, P., Harpham, Q. and Hummel, S. 2016. FluidEarth, https://sourceforge.net/projects/fluidearth/, accessed $3^{\text {rd }}$ April 2019. 
Harpham, Q. K., Cleverley, P. \& Kelly, D. 2104 The Fluid Earth 2 implementation of OpenMI 2.0. J. Hydroinform. 16(4), 890-906.

Harpham, Q., Lhomme, J., Parodi, A., Fiori, E., Jagers, B. and Galizia, A. 2016. Using OpenMI and a Model MAP to Integrate WaterML2 and NetCDF Data Sources into Flood Modeling of Genoa, Italy. JAWRA Journal of the American Water Resources Association (2016).

Harpham, Q.K. 2018. Using spatio-temporal feature type structures for coupling environmental numerical models to each other and to data sources, under review for publication, Open University, Milton Keynes, UK.

Harpham, Q. and Danovaro, E., 2015. Towards standard metadata to support models and interfaces in a hydro-meteorological model chain. Journal of Hydroinformatics, 17(2), pp.260-274.

Hughes, A. G., Mansour, M. M., \& Robins, N. S. 2008. Evaluation of distributed recharge in an upland semi-arid karst system: the West Bank Mountain Aquifer, Middle East. Hydrogeology Journal, 16(5), 845-854.

Iwanaga, T., Rahman, J., Partington, D., Croke, B. and Jakeman, A. 2018. 'Software Development Practices in Integrated Environmental Model Development', Proc. 9th International Congress on Environmental Modelling and Software, Fort Collins, Colorado, USA. Mazdak Arabi, Olaf David, Jack Carlson, Daniel P. Ames (Eds.).

Jackson C.R.; Spink A.E.F., 2004. User's manual for the groundwater flow model ZOOMQ3D. Nottingham, UK, British Geological Survey, 107pp. (IR/04/140) - http://nora.nerc.ac.uk/11829/

Jamieson, S., Lhomme, J., Wright, G. and Gouldby, B. 2012. Highly efficient 2D inundation modelling with enhanced diffusion-wave and sub-element topography. Proc. Inst. Civ. Eng. Wat. Man. 165 (10): 581-595.

Laniak, G.F., Olchin, G., Goodall, J., Voinov, A., Hill, M., Glynn, P., Whelan, G., Geller, G., Quinn, N., Blind, M. and Peckham, S., 2013. Integrated environmental modeling: a vision and roadmap for the future. Environmental Modelling \& Software, 39, pp.3-23.

Mansour, M.M.; Hughes, A.G. 2004 User's manual for the distributed recharge model ZOODRM. British Geological Survey, 61pp. (IR/04/150) - nora.nerc.ac.uk/12633/

Mansour, M., Mackay, J., Abesser, C., Williams, A., Wang, L., Bricker, S., \& Jackson, C. 2013. Integrated Environmental Modeling applied at the basin scale: linking different types of models using the OpenMI standard to improve simulation of groundwater processes in the Thames Basin, UK. In: MODFLOW and More 2013: Translating Science into Practice, Colorado, USA, 2-5 June 2013. (nora.nerc.ac.uk/501789/)

Meiburg, S. in EPA 2008 Integrated Modeling for Integrated Environmental Decision Making. EPA100-R-08-010. US Environmental Protection Agency, Office of the Science Advisor, Washington, DC.

Moore, R. V., \& Hughes, A. G., 2017. Integrated environmental modelling: achieving the vision. Geological Society, London, Special Publications, 408(1), 17-34.

Nativi, S., Mazzetti, P., \& Geller, G. N., 2013. Environmental model access and interoperability: The GEO Model Web initiative. Environmental Modelling \& Software, 39, 214-228. 
OGC OpenMI. 2014. OGC Open Modelling Interface - Interface Standard OGC 11---014r3 version 2.0. Open Geospatial Consortium, http://www.opengis.net/doc/IS/openmi/2.0GC, accessed $26^{\text {th }}$ February 2019.

Ramsbottom D., Floyd P. and Penning-Rowsell E. 2003. Flood Risks to People Phase 1 R\&D Technical Report FD2317, ISBN 1844321355.

Royse, K. R., de Freitas, M., Burgess, W. G., Cosgrove, J., Ghail, R. C., Gibbard, P., ... \& Skipper, J. (2012). Geology of London, UK. Proceedings of the Geologists' Association, 123(1), 22-45.

Sophocleous, M. A., Koelliker, J. K., Govindaraju, R. S., Birdie, T., Ramireddygari, S. R., \& Perkins, S. P., 1999. Integrated numerical modeling for basin-wide water management: The case of the Rattlesnake Creek basin in south-central Kansas. Journal of Hydrology, 214(1), 179-196.

Voinov, A. 2010 Model integration and the role of data. Environmental Modelling \& Software 25, 965-969. 